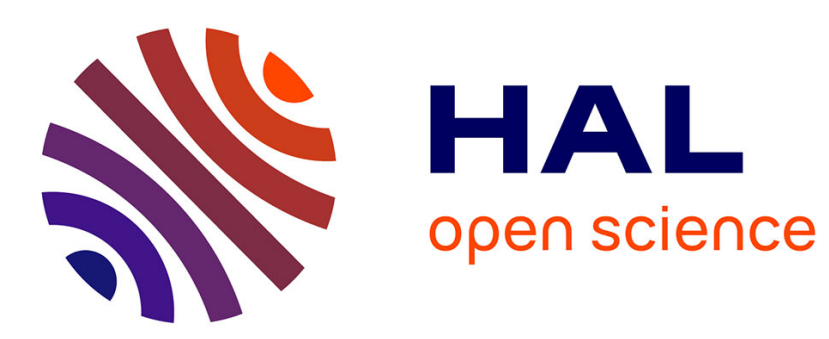

\title{
On stationary solutions to normal, coplanar discrete Boltzmann equation models
}

\author{
Leif Arkeryd, Anne Nouri
}

\section{To cite this version:}

Leif Arkeryd, Anne Nouri. On stationary solutions to normal, coplanar discrete Boltzmann equation models. 2020. hal-02520761

\author{
HAL Id: hal-02520761 \\ https://hal.science/hal-02520761 \\ Preprint submitted on 26 Mar 2020
}

HAL is a multi-disciplinary open access archive for the deposit and dissemination of scientific research documents, whether they are published or not. The documents may come from teaching and research institutions in France or abroad, or from public or private research centers.
L'archive ouverte pluridisciplinaire HAL, est destinée au dépôt et à la diffusion de documents scientifiques de niveau recherche, publiés ou non, émanant des établissements d'enseignement et de recherche français ou étrangers, des laboratoires publics ou privés. 


\title{
On stationary solutions to normal, coplanar discrete Boltzmann equation models.
}

\author{
Leif ARKERYD and Anne NOURI \\ Mathematical Sciences, 41296 Göteborg, Sweden, \\ arkeryd@chalmers.se \\ Aix-Marseille University, CNRS, Centrale Marseille, I2M UMR 7373, 13453 Marseille, France, \\ anne.nouri@univ-amu.fr
}

\begin{abstract}
The paper proves existence of renormalized solutions for a class of velocity-discrete coplanar stationary Boltzmann equations with given indata. The proof is based on the construction of a sequence of approximations with $L^{1}$ compactness for the integrated collision frequency and gain term. $L^{1}$ compactness of a sequence of approximations is obtained using the Kolmogorov-Riesz theorem and replaces the $L^{1}$ compactness of velocity averages in the continuous velocity case, not available when the velocities are discrete.
\end{abstract}

\section{Introduction.}

The Boltzmann equation is the fundamental mathematical model in the kinetic theory of gases. Replacing its continuum of velocities with a discrete set of velocites is a simplification preserving the essential features of free flow and quadratic collision term. Besides this fundamental aspect they can approximate the Boltzmann equation with any given accuracy [4], and are thereby useful for approximations and numerics. In the quantum realm they can also be more directly connected to microscopic quasi/particle models.

A discrete velocity model of a kinetic gas, is a system of partial differential equations having the form,

$$
\frac{\partial f_{i}}{\partial t}(t, z)+v_{i} \cdot \nabla_{z} f_{i}(t, z)=Q_{i}(f)(t, z), \quad t>0, \quad z \in \Omega, \quad 1 \leq i \leq p,
$$

where $f_{i}, 1 \leq i \leq p$, are phase space densities at time $t$, position $z$, velocity $v_{i}, \Omega \subset \mathbb{R}^{d}$, and $v_{i} \in \mathbb{R}^{d}$, $1 \leq i \leq p$, are given discrete velocities. The collision operator $Q=\left(Q_{i}\right)_{1 \leq i \leq p}$ with gain part $Q^{+}$, loss part $Q^{-}$, and collision frequency $\nu$, is given by

$$
\begin{aligned}
Q_{i}(f) & =\sum_{j, k, l=1}^{p} \Gamma_{i j}^{k l}\left(f_{k} f_{l}-f_{i} f_{j}\right) \\
& =Q_{i}^{+}(f)-Q_{i}^{-}(f), \quad Q_{i}^{-}(f)=f_{i} \nu_{i}(f), \quad i=1, \ldots, p .
\end{aligned}
$$

\footnotetext{
${ }^{1} 2010$ Mathematics Subject Classification; 60K35, 82C40, 82C99.

${ }^{2}$ Key words; stationary Boltzmann equation, discrete coplanar velocities, normal model, entropy.
} 
The collision coefficients satisfy

$$
\Gamma_{i j}^{k l}=\Gamma_{j i}^{k l}=\Gamma_{k l}^{i j} \geq 0 .
$$

If a collision coefficient $\Gamma_{i j}^{k l}$ is non-zero, then the conservation laws for momentum and energy,

$$
v_{i}+v_{j}=v_{k}+v_{l}, \quad\left|v_{i}\right|^{2}+\left|v_{j}\right|^{2}=\left|v_{k}\right|^{2}+\left|v_{l}\right|^{2},
$$

are satisfied. The discrete velocity model (DVM) is called normal (see [5]) if any solution of the equations

$$
\Psi\left(v_{i}\right)+\Psi\left(v_{j}\right)=\Psi\left(v_{k}\right)+\Psi\left(v_{l}\right)
$$

where the indices $(i, j ; k, l)$ take all possible values satisfying $\Gamma_{i j}^{k l}>0$, is given by

$$
\Psi(v)=a+b \cdot v+c|v|^{2}
$$

for some constants $a, c \in \mathbb{R}$ and $b \in \mathbb{R}^{d}$. This paper studies stationary solutions to coplanar models, i.e. with $v_{i} \in \mathbb{R}^{2}, 1 \leq i \leq p$, in a strictly convex bounded open subset $\Omega \subset \mathbb{R}^{2}$, with $C^{1}$ boundary $\partial \Omega$ and given indata. We consider

the generic situation of normal coplanar velocities with no pair of velocities $v_{i}, v_{j}, 1 \leq i, j \leq p$, parallel,

and additionally that for some direction $n_{0} \in \mathbb{R}^{2}, \quad v_{i} \cdot n_{0}>0, \quad 1 \leq i \leq p$.

For stationary solutions to the Broadwell model, that does not belong to this class, see [2], [6].

Denote by $n(Z)$ the inward normal to $Z \in \partial \Omega$. Denote the $v_{i}$-ingoing (resp. $v_{i}$-outgoing) part of the boundary by

$$
\partial \Omega_{i}^{+}=\left\{Z \in \partial \Omega ; v_{i} \cdot n(Z)>0\right\}, \quad\left(\text { resp. } \partial \Omega_{i}^{-}=\left\{Z \in \partial \Omega ; v_{i} \cdot n(Z)<0\right\}\right) .
$$

Let

$$
s_{i}^{+}(z)=\inf \left\{s>0 ; z-s v_{i} \in \partial \Omega_{i}^{+}\right\}, \quad s_{i}^{-}(z)=\inf \left\{s>0 ; z+s v_{i} \in \partial \Omega_{i}^{-}\right\}, \quad z \in \Omega .
$$

Write

$$
z_{i}^{+}(z)=z-s_{i}^{+}(z) v_{i} \quad\left(\operatorname{resp} . z_{i}^{-}(z)=z+s_{i}^{-}(z) v_{i}\right)
$$

for the ingoing (resp. outgoing) point on $\partial \Omega$ of the characteristics through $z$ in direction $v_{i}$. The boundary value problem

$$
\begin{aligned}
& v_{i} \cdot \nabla f_{i}(z)=Q_{i}(f)(z), \quad z \in \Omega, \\
& f_{i}(z)=f_{b i}(z), \quad z \in \partial \Omega_{i}^{+}, \quad 1 \leq i \leq p,
\end{aligned}
$$

is considered in $L^{1}$ in one of the following equivalent forms;

the exponential multiplier form,

$$
\begin{aligned}
f_{i}(z) & =f_{b i}\left(z_{i}^{+}(z)\right) e^{-\int_{0}^{s_{i}^{+}(z)} \nu_{i}(f)\left(z_{i}^{+}(z)+s v_{i}\right) d s} \\
& +\int_{0}^{s_{i}^{+}(z)} Q_{i}^{+}(f)\left(z_{i}^{+}(z)+s v_{i}\right) e^{-\int_{s}^{s_{i}^{+}(z)} \nu_{i}(f)\left(z_{i}^{+}(z)+r v_{i}\right) d r} d s, \quad \text { a.a. } z \in \Omega, \quad 1 \leq i \leq p,
\end{aligned}
$$


the mild form,

$$
f_{i}(z)=f_{b i}\left(z_{i}^{+}(z)\right)+\int_{0}^{s_{i}^{+}(z)} Q_{i}(f)\left(z_{i}^{+}(z)+s v_{i}\right) d s, \quad \text { a.a. } z \in \Omega, \quad 1 \leq i \leq p,
$$

the renormalized form,

$$
v_{i} \cdot \nabla \ln \left(1+f_{i}\right)(z)=\frac{Q_{i}(f)}{1+f_{i}}(z), \quad z \in \Omega, \quad f_{i}(z)=f_{b i}(z), \quad z \in \partial \Omega_{i}^{+}, \quad 1 \leq i \leq p,
$$

in the sense of distributions.

Denote by $L_{+}^{1}(\Omega)$ the set of non-negative integrable functions on $\Omega$. The main result of the present paper is

\section{Theorem 1.1}

Consider a coplanar collision operator in the generic case of (1.3) additionally satisfying (1.4), and non-negative ingoing boundary values $f_{b i}, 1 \leq i \leq p$, with mass and entropy bounded,

$$
\int_{\partial \Omega_{i}^{+}} v_{i} \cdot n(z) f_{b i}\left(1+\ln f_{b i}\right)(z) d \sigma(z)<+\infty, \quad 1 \leq i \leq p .
$$

There exists a stationary renormalized solution in $\left(L_{+}^{1}(\Omega)\right)^{p}$ to the boundary value problem (1.6)(1.7) with finite entropy-dissipation.

Most mathematical results for stationary discrete velocity models of the Boltzmann equation have been obtained in one space dimension. An overview is given in [8]. In two dimensions, special classes of solutions to the Broadwell model are given in [6], [3], and [9]. The Broadwell model is a four-velocity model, with $v_{1}+v_{2}=v_{3}+v_{4}=0$ and $v_{1}, v_{2}$ orthogonal. [6] contains a detailed study of the stationary Broadwell equation in a rectangle with comparison to a Carleman-like system, and a discussion of (in)compressibility aspects. A main result in [6] is the existence of continuous solutions to the two-dimensional stationary Broadwell model with continuous boundary data for a rectangle. The proof starts by solving the problem with a given gain term, and uses the compactness of the corresponding twice iterated solution operator to conclude by Schaeffer's fixed point theorem. The paper [2] studies that problem in an $L^{1}$-setting, with the proof broadly within the frame of the present paper. In both those papers of ours, there is a priori control of mass and entropy dissipation. Denoting by $f_{i}(t, \cdot), 1 \leq i \leq 4$, the density of the particles moving with velocity $v_{i}$ at time $t$, the proof in [2] in an essential way uses the constancy of the sums $f_{1}+f_{2}$ and $f_{3}+f_{4}$ along characteristics, which no longer holds in this paper. It is here replaced by a compactness property for the collision frequency and gain parts in the exponential form of the approximations employed. The compactness is based on assumption (1.3) and the simultaneous presence of space integrals in two velocity directions.

The proof starts from bounded approximations with damping and convolution added, written in exponential multiplyer form, and solved by a fixed point argument. Then the damping and convolutions are removed by taking limits using $L^{1}$-compactness of the integrated collision frequency and gain term. The compactness is proven by the Kolmogorov-Riesz theorem (see [10], [11]). The limit of the remaining approximations is obtained by using again the Kolmogorov-Riesz theorem.

\section{Approximations.}

The construction of the primary approximated boundary value problem with damping and convolutions is similar to the Broadwell case [2]. Denote $a \wedge b$ the minimum of two real numbers $a$ and 
b. Take $\alpha>0$ and set

$$
c_{\alpha}=\frac{1}{\alpha} \sum_{i=1}^{p} \int_{\partial \Omega_{i}^{+}}\left(n(z) \cdot v_{i}\right) f_{b i}(z) d \sigma(z), \quad K_{\alpha}=\left\{f \in\left(L_{+}^{1}(\Omega)\right)^{p} ; \sum_{i=1}^{p} \int_{\Omega} f_{i}(z) d z \leq c_{\alpha}\right\} .
$$

Let $\mu_{\alpha}$ be a smooth mollifier in $\mathbb{R}^{2}$ with support in the ball centered at the origin of radius $\alpha$. Outside the boundary the function to be convolved with $\mu_{\alpha}$ is continued in the normal direction by its boundary value. Let $\tilde{\mu}_{k}$ be a smooth mollifier on $\partial \Omega$. Denote by

$$
f_{b i}^{k}=\left(f_{b i}(\cdot) \wedge \frac{k}{2}\right) * \tilde{\mu}_{k}, \quad 1 \leq i \leq p .
$$

Let $\mathcal{T}$ be the map defined on $K_{\alpha}$ by $\mathcal{T}(f)=F$, where $F=\left(F_{i}\right)_{1 \leq i \leq p}$ is the solution of

$$
\begin{aligned}
& \alpha F_{i}+v_{i} \cdot \nabla F_{i}=\sum_{j, l, m=1}^{p} \Gamma_{i j}^{l m}\left(\frac{F_{l}}{1+\frac{F_{l}}{k}} \frac{f_{m} * \mu_{\alpha}}{1+\frac{f_{m} * \mu_{\alpha}}{k}}-\frac{F_{i}}{1+\frac{F_{i}}{k}} \frac{f_{j} * \mu_{\alpha}}{1+\frac{f_{j} * \mu_{\alpha}}{k}}\right), \\
& F_{i}\left(z_{i}^{+}(z)\right)=f_{b i}^{k}\left(z_{i}^{+}(z)\right) .
\end{aligned}
$$

$F=\mathcal{T}(f)$ can be obtained as the limit in $\left(L_{+}^{1}(\Omega)\right)^{p}$ of the sequence $\left(F^{q}\right)_{q \in \mathbb{N}}$ defined by $F^{0}=0$ and

$$
\begin{aligned}
& \alpha F_{i}^{q+1}+v_{i} \cdot \nabla F_{i}^{q+1}=\sum_{j, l, m=1}^{p} \Gamma_{i j}^{l m}\left(\frac{F_{l}^{q}}{1+\frac{F_{l}^{q}}{k}} \frac{f_{m} * \mu_{\alpha}}{1+\frac{f_{m} * \mu_{\alpha}}{k}}-\frac{F_{i}^{q+1}}{1+\frac{F_{i}^{q}}{k}} \frac{f_{j} * \mu_{\alpha}}{1+\frac{f_{j} * \mu_{\alpha}}{k}}\right), \\
& F_{i}^{q+1}\left(z_{i}^{+}(z)\right)=f_{b i}^{k}\left(z_{i}^{+}(z)\right), \\
& q \in \mathbb{N} .
\end{aligned}
$$

The sequence $\left(F^{q}\right)_{q \in \mathbb{N}}$ is monotone. Indeed,

$$
F_{i}^{0} \leq F_{i}^{1}, \quad 1 \leq i \leq n
$$

by the exponential form of $F_{i}^{1}$. If $F_{i}^{q} \leq F_{i}^{q+1}, 1 \leq i \leq p$, then it follows from the exponential form that $F_{i}^{q+1} \leq F_{i}^{q+2}$. Moreover,

$$
\alpha \sum_{i=1}^{p} F_{i}^{q+1}+\sum_{i=1}^{p} v_{i} \cdot \nabla F_{i}^{q+1}=\sum_{i, j, l, m=1}^{p} \Gamma_{i j}^{l m} \frac{\left(F_{l}^{q}-F_{l}^{q+1}\right)}{1+\frac{F_{l}^{p}}{k}} \frac{f_{m} * \mu_{\alpha}}{1+\frac{f_{m} * \mu_{\alpha}}{k}} \leq 0,
$$

so that

$$
\sum_{i=1}^{p} \int_{\Omega} F_{i}^{q+1}(z) d z \leq c_{\alpha}
$$

By the monotone convergence theorem, $\left(F^{q}\right)_{q \in \mathbb{N}}$ converges in $L^{1}(\Omega)$ to a solution $F$ of $(2.2)-(2.3)$. The solution of (2.2)-(2.3) is unique in the set of non-negative functions. Indeed, let $G=\left(G_{i}\right)_{1 \leq i \leq p}$ be a non-negative solution of (2.2)-(2.3). It follows by induction that

$$
\forall q \in \mathbb{N}, \quad F_{i}^{q} \leq G_{i}, \quad 1 \leq i \leq p .
$$

Indeed, (2.7) holds for $q=0$, since $G_{i} \geq 0,1 \leq i \leq p$. Assume (2.7) holds for $q$. Using the exponential form of $F_{i}^{q+1}$ implies $F_{i}^{q+1} \leq G_{i}$. Consequently,

$$
F_{i} \leq G_{i}, \quad 1 \leq i \leq p
$$


Moreover, subtracting the partial differential equations satisfied by $G_{i}$ from the partial differential equations satisfied by $F_{i}, 1 \leq i \leq p$, and integrating the resulting equation on $\Omega$, it results

$$
\alpha \sum_{i=1}^{p} \int_{\Omega}\left(G_{i}-F_{i}\right)(z) d z+\sum_{i=1}^{p} \int_{\partial \Omega_{i}^{-}}\left|n(z) \cdot v_{i}\right|\left(G_{i}-F_{i}\right)(z) d \sigma(z)=0 .
$$

It results from (2.8)-(2.9) that $G=F$.

The map $\mathcal{T}$ is continuous in the $L^{1}$-norm topology (cf [1] pages 124-5). Namely, let a sequence $\left(f^{q}\right)_{q \in \mathbb{N}}$ in $K_{\alpha}$ converge in $\left(L^{1}(\Omega)\right)^{p}$ to $f \in K_{\alpha}$. Set $F^{q}=\mathcal{T}\left(f^{q}\right)$. Because of the uniqueness of the solution to (2.2)-(2.3), it is enough to prove that there is a subsequence of $\left(F^{q}\right)$ converging to $F=\mathcal{T}(f)$. Now there is a subsequence of $\left(f^{q}\right)$, still denoted $\left(f^{q}\right)$, such that decreasingly (resp. increasingly) $\left(G^{q}\right)=\left(\sup _{r \geq q} f^{r}\right)$ (resp. $\left.\left(g^{q}\right)=\left(\inf _{r \geq q} f^{r}\right)\right)$ converges to $f$ in $L^{1}$. Let $\left(S^{q}\right)$ (resp. $\left.\left(s^{q}\right)\right)$ be the sequence of solutions to

$$
\begin{aligned}
& \alpha S_{i}^{q}+v_{i} \cdot \nabla S_{i}^{q}=\sum_{j, l, m=1}^{p} \Gamma_{i j}^{l m}\left(\frac{S_{l}^{q}}{1+\frac{S_{l}^{q}}{k}} \frac{G_{m}^{q} * \mu_{\alpha}}{1+\frac{G_{m}^{q} * \mu_{\alpha}}{k}}-\frac{S_{i}^{q}}{1+\frac{S_{i}^{q}}{k}} \frac{g_{j}^{q} * \mu_{\alpha}}{1+\frac{g_{j}^{q} * \mu_{\alpha}}{k}}\right) \\
& S_{i}^{q}\left(z_{i}^{+}(z)\right)=f_{b i}^{k}\left(z_{i}^{+}(z)\right) \\
& \alpha s_{i}^{q}+v_{i} \cdot \nabla_{x} s_{i}^{q}=\sum_{j, l, m=1}^{p} \Gamma_{i j}^{l m}\left(\frac{s_{l}^{q}}{1+\frac{s_{l}^{q}}{k}} \frac{g_{m}^{q} * \mu_{\alpha}}{1+\frac{g_{m}^{q} * \mu_{\alpha}}{k}}-\frac{s_{i}^{q}}{1+\frac{s_{i}^{q}}{k}} \frac{G_{j}^{q} * \mu_{\alpha}}{1+\frac{G_{j}^{q} * \mu_{\alpha}}{k}}\right) \\
& s_{i}^{q}\left(z_{i}^{+}(z)\right)=f_{b i}^{k}\left(z_{i}^{+}(z)\right) .
\end{aligned}
$$

$\left(S^{q}\right)$ is a non-increasing sequence, since that holds for the successive iterates defining the sequence. Then $\left(S^{q}\right)$ decreasingly converges in $L^{1}$ to some $S$. Similarly $\left(s^{q}\right)$ increasingly converges in $L^{1}$ to some $s$. The limits $S$ and $s$ satisfy (2.2)-(2.3). It follows by uniqueness that $s=F=S$, hence that $\left(F^{q}\right)$ converges in $L^{1}$ to $F$.

The map $\mathcal{T}$ is also compact in the $L^{1}$-norm topology. Indeed, let $\left(f^{q}\right)_{q \in \mathbb{N}}$ be a sequence in $K_{\alpha}$ and $\left(F^{q}\right)_{q \in \mathbb{N}}=\left(\mathcal{T}\left(f^{q}\right)\right)_{q \in \mathbb{N}}$. The boundedness by $k^{2}$ of the terms in the collision operator, induces uniform $L^{1}$ equi-continuity of $\left(F_{i}^{q}\right)_{q \in \mathbb{N}}$ with respect to the $v_{i}$-direction, as follows from the mild form of the equations. For the uniform $L^{1}$ equi-continuity with respect to the $v_{j}$-direction, $j \neq i$, consider for each $q$ and with $f:=f^{q}$ the sequence $\left(G^{q, r}\right)_{r \in \mathbb{N}}$ defined by $G^{q, 0}=0$ and for $r \in \mathbb{N}^{*}$

$$
\begin{aligned}
& \alpha G_{i}^{q, r}+v_{i} \cdot \nabla G_{i}^{q, r}=\sum_{j, l, m=1}^{p} \Gamma_{i j}^{l m}\left(\frac{G_{l}^{q, r}}{1+\frac{G_{l}^{q, r}}{k}} \frac{f_{m} * \mu_{\alpha}}{1+\frac{f_{m} * \mu_{\alpha}}{k}}-\frac{G_{i}^{q, r}}{1+\frac{G_{i}^{q, r-1}}{k}} \frac{f_{j} * \mu_{\alpha}}{1+\frac{f_{j} * \mu_{\alpha}}{k}}\right), \\
& \left.G_{i}^{q, r}\left(z_{i}^{+}(z)\right)=f_{b i}^{k}\left(z_{i}^{+}(z)\right)\right), \quad 1 \leq i \leq p .
\end{aligned}
$$

The existence of a unique solution for each $r$ follows as for the problem (2.2)-(2.3). By induction on $r$, prove that $\left(G^{q, r}\right)_{q \in \mathbb{N}}$ is uniformly equicontinuous in the $v_{j}$-direction. It holds for $r=0$. Assume it holds for $r-1 \in \mathbb{N}^{*}$ and prove it for $r$. Writing $G^{q, r}(z)$ in exponential form and using the uniform equicontinuity in the $v_{j}$-direction of $\left(G^{q, r-1}\right)_{q \in \mathbb{N}}$ and the compactness of $\left(f^{q} * \mu_{\alpha}\right)$, it comes back to prove the uniform equicontinuity in the $v_{j}$-direction of

$$
z \rightarrow \int_{0}^{s_{i}\left(z_{i}^{-}(z)\right)} \frac{G_{l}^{q, r}}{1+\frac{G_{l}^{q, r}}{k}}\left(z_{i}^{-}(z)+s v_{i}\right) d s
$$


First,

$$
z_{i}^{-}\left(z+h v_{j}\right)=z_{i}^{-}(z)+a v_{i}+b v_{l}, \quad \text { with } \quad \lim _{h \rightarrow 0} a(h)=\lim _{h \rightarrow 0} b(h)=0,
$$

uniformly with respect to $z \in \Omega$. Consequently,

$$
\begin{aligned}
& \int\left|\int_{0}^{s_{i}\left(z_{i}^{-}(z)\right)} \frac{G_{l}^{q, r}}{1+\frac{G_{l}^{q, r}}{k}}\left(z_{i}^{-}\left(z+h v_{j}\right)+s v_{i}\right)-\frac{G_{l}^{q, r}}{1+\frac{G_{l}^{q, r}}{k}}\left(z_{i}^{-}(z)+s v_{i}\right) d s\right| d z \\
& \leq \iint_{0}^{s_{i}\left(z_{i}^{-}(z)\right)}\left|\frac{G_{l}^{q, r}}{1+\frac{G_{l}^{q, r}}{k}}\left(z_{i}^{-}(z)+(a+s) v_{i}+b v_{l}\right)-\frac{G_{l}^{q, r}}{1+\frac{G_{l}^{q, r}}{k}}\left(z_{i}^{-}(z)+(a+s) v_{i}\right)\right| d s d z \\
& +\int\left|\int_{0}^{s_{i}\left(z_{i}^{-}(z)\right)} \frac{G_{l}^{q, r}}{1+\frac{G_{l}^{q, r}}{k}}\left(z_{i}^{-}(z)+(a+s) v_{i}\right)-\frac{G_{l}^{q, r}}{1+\frac{G_{l}^{q, r}}{k}}\left(z_{i}^{-}(z)+s v_{i}\right) d s\right| d z .
\end{aligned}
$$

The limit when $h \rightarrow 0$ of $(2.16)$ is zero, by the uniform $L^{1}$ equicontinuity of $\left(G_{l}^{q, r}\right)_{q \in \mathbb{N}}$ with respect to the $v_{l}$-direction. With the change of variables $s \rightarrow a+s$ in its first integral, (2.17) equals

$$
\int\left|\int_{s_{i}\left(z_{i}^{-}(z)\right)}^{s_{i}\left(z_{i}^{-}(z)\right)+a} \frac{G_{l}^{q, r}}{1+\frac{G_{l}^{q, r}}{k}}\left(z_{i}^{-}(z)+s v_{i}\right) d s-\int_{0}^{a} \frac{G_{l}^{q, r}}{1+\frac{G_{l}^{q, r}}{k}}\left(z_{i}^{-}(z)+s v_{i}\right) d s\right| d z,
$$

which tends to zero when $h$ tends to zero since both integrands are bounded by $k$. This proves the $L^{1}$ compactness of $\left(G_{i}^{q, r}\right)_{q \in \mathbb{N}}$. For $q$ fixed, the sequence $\left(G_{i}^{q, r}\right)_{r \in \mathbb{N}}$ is increasing, and its limit satisfies (2.2)-(2.3) with $f=f^{q}$, so the limit equals $F_{i}^{q}$. Take a subsequence of $q$ still denoted by $q$, with $\left(f^{q} * \mu_{\alpha}\right)$ convergent in $L^{1}$ to some $f^{\infty}$ when $q \rightarrow \infty$, and a further subsequence so that $\left(G^{q, 1}\right)$ converges to some $F^{\infty, 1}$ in $L^{1}$. Continue by diagonalization to convergence of $\left(G^{q, r}\right)_{q}$ to $F^{\infty, r}$ for all $r \in \mathbb{N}$. The limits satisfy (2.14)-(2.15) with $f * \mu_{\alpha}$ replaced with $f^{\infty}$, and $G^{q, r}$ with $F^{\infty, r}$ giving an increasing sequence, with limit satisfying (2.2)-(2.3), where $f * \mu_{\alpha}$ is replaced with $f^{\infty}$. So given a sequence in $K_{\alpha}$, there is a subsequence with converging image under $\mathcal{T}$. The compactness of $\mathcal{T}$ is thus proved.

Hence by the Schauder fixed point theorem,

Lemma 2.1 There is a fixed point in $\left(L_{+}^{1}(\Omega)\right)^{p}$ to $\mathcal{T}$ i.e. a solution $F \in\left(L_{+}^{1}(\Omega)\right)^{p}$ to

$$
\begin{aligned}
& \alpha F_{i}+v_{i} \cdot \nabla F_{i}=\sum_{j, l, m=1}^{p} \Gamma_{i j}^{l m}\left(\frac{F_{l}}{1+\frac{F_{l}}{k}} \frac{F_{m} * \mu_{\alpha}}{1+\frac{F_{m} * \mu_{\alpha}}{k}}-\frac{F_{i}}{1+\frac{F_{i}}{k}} \frac{F_{j} * \mu_{\alpha}}{1+\frac{F_{j} * \mu_{\alpha}}{k}}\right), \\
& F_{i}\left(z_{i}^{+}(z)\right)=f_{b i}^{k}\left(z_{i}^{+}(z)\right), \quad 1 \leq i \leq p .
\end{aligned}
$$

\section{Removal of the damping and convolutions.}

Let $k>1$ be fixed. Denote by $F^{\alpha}$ the solution to (2.19)-(2.20) obtained in the previous section. Each component of $F^{\alpha}$ being bounded by a multiple of $k^{2},\left(F^{\alpha}\right)_{\alpha \in] 0,1[}$ is weakly compact in $\left(L^{1}(\Omega)\right)^{p}$. Denote by $F^{k}$ the limit for the weak topology in $\left(L^{1}(\Omega)\right)^{p}$ of a converging subsequence when $\alpha \rightarrow 0$. Let us prove that for a subsequence, the convergence is strong in $\left(L^{1}(\Omega)\right)^{p}$.

Lemma 3.1 There is a sequence $\left(\alpha_{q}\right)_{q \in \mathbb{N}}$ tending to zero when $q \rightarrow+\infty$, such that $\left(F^{\alpha_{q}}\right)_{q \in \mathbb{N}}$ strongly converges to $F^{k}$ in $\left(L^{1}(\Omega)\right)^{p}$ when $q \rightarrow+\infty$. 
Proof of Lemma 3.1

Consider the approximation scheme $\left(f^{\alpha, \kappa}\right)_{\kappa \in \mathbb{N}}$ of $F^{\alpha}$,

$$
\begin{aligned}
& f_{i}^{\alpha, 0}=0, \\
& \alpha f_{i}^{\alpha, \kappa+1}+v_{i} \cdot \nabla f_{i}^{\alpha, \kappa+1}=\sum_{j, l, m=1}^{p} \Gamma_{i j}^{l m}\left(\frac{F_{l}^{\alpha}}{1+\frac{F_{l}^{\alpha}}{k}} \frac{F_{m}^{\alpha} * \mu_{\alpha}}{1+\frac{F_{m}^{\alpha} * \mu_{\alpha}}{k}}-\frac{f_{i}^{\alpha, \kappa+1}}{1+\frac{f_{i}^{\alpha, \kappa+1}}{k}} \frac{f_{j}^{\alpha, \kappa} * \mu_{\alpha}}{1+\frac{f_{j}^{\alpha, \kappa} * \mu_{\alpha}}{k}}\right), \\
& f_{i}^{\alpha, \kappa+1}\left(z_{i}^{+}(z)\right)=f_{b i}^{k}\left(z_{i}^{+}(z)\right), \quad 1 \leq i \leq p, \quad \kappa \in \mathbb{N} .
\end{aligned}
$$

$f^{\alpha, 1}$ is obviously given in terms of $F^{\alpha}$. It follows from the exponential form that $\left.F_{i}^{\alpha} \leq f_{i}^{\alpha, 1}, \alpha \in\right] 0,1[$. Denote by $\mathcal{S}$ the map from $\mathbb{R}^{p} \times \mathbb{R}^{p}$ mapping $(X, Z)$ into $W=\mathcal{S}(X, Z) \in \mathbb{R}^{p}$ solution to

$$
\begin{aligned}
& \alpha W_{i}+v_{i} \cdot \nabla W_{i}=\sum_{j, l, m=1}^{p} \Gamma_{i j}^{l m}\left(\frac{F_{l}^{\alpha}}{1+\frac{F_{l}^{\alpha}}{k}} \frac{F_{m}^{\alpha} * \mu_{\alpha}}{1+\frac{F_{m}^{\alpha} * \mu_{\alpha}}{k}}-\frac{W_{i}}{1+\frac{X_{i}}{k}} \frac{Z_{j} * \mu_{\alpha}}{1+\frac{Z_{j} * \mu_{\alpha}}{k}}\right) \\
& W_{i}\left(z_{i}^{+}(z)\right)=f_{b i}^{k}\left(z_{i}^{+}(z)\right), \quad 1 \leq i \leq p .
\end{aligned}
$$

Denote by

$$
\begin{aligned}
& f^{\alpha, 1,0}=\mathcal{S}\left(0, f^{\alpha, 1}\right), \quad f^{\alpha, 1, r}=\mathcal{S}\left(f^{\alpha, 1, r-1}, f^{\alpha, 1}\right), \\
& F^{\alpha, 0}=\mathcal{S}\left(0, F^{\alpha}\right), \quad F^{\alpha, r}=\mathcal{S}\left(F^{\alpha, r-1}, F^{\alpha}\right), \quad r \in \mathbb{N}^{*}
\end{aligned}
$$

First,

$$
f_{i}^{\alpha, 1,0} \leq F_{i}^{\alpha, 0}
$$

Then the sequence $\left(f_{i}^{\alpha, 1, r}\right)_{r \in \mathbb{N}}\left(\right.$ resp. $\left.\left(F_{i}^{\alpha, r}\right)_{r \in \mathbb{N}}\right)$ is increasing with limit $f_{i}^{\alpha, 2}$ (resp. $F_{i}^{\alpha}$ ). It follows from $f_{i}^{\alpha, 1, r} \leq F_{i}^{\alpha, r}, r \in \mathbb{N}$, that

$$
f_{i}^{\alpha, 2} \leq F_{i}^{\alpha}, \quad 1 \leq i \leq p
$$

Let

$$
f^{\alpha, 2,0}:=\mathcal{S}\left(0, f^{\alpha, 2}\right), \quad f^{\alpha, 2, r}:=\mathcal{S}\left(f^{\alpha, r-1}, f^{\alpha, 2}\right), \quad r \in \mathbb{N}^{*} .
$$

It follows from (3.4) that

$$
f_{i}^{\alpha, 2,0} \geq F_{i}^{\alpha, 0}, \quad 1 \leq i \leq p
$$

The sequence $\left(f_{i}^{\alpha, 2, r}\right)_{r \in \mathbb{N}}$ is also increasing with limit $f_{i}^{\alpha, 3}$ and with $f_{i}^{\alpha, 2, r} \geq F_{i}^{\alpha, r}$. Hence

$$
f_{i}^{\alpha, 3} \geq F_{i}^{\alpha}
$$

From here by induction on $\kappa$, it holds that

$$
\left.f_{i}^{\alpha, 2 \kappa} \leq f_{i}^{\alpha, 2 \kappa+2} \leq F_{i}^{\alpha} \leq f_{i}^{\alpha, 2 \kappa+3} \leq f_{i}^{\alpha, 2 \kappa+1}, \quad \alpha \in\right] 0,1[, \quad \kappa \in \mathbb{N} .
$$

By induction on $r$, for each $r$ the sequence $\left(f^{\alpha, 1, r}\right)_{\alpha \in] 0,1[}$ is translationally equicontinuous in $\alpha$. The limit sequence $\left(f^{\alpha, 2}\right)_{\alpha \in] 0,1[}$ is also translationally equicontinuous. This is so, since given $\epsilon>0, r$ and then $h_{0}$ can be taken so that

$$
\int\left(f^{\alpha, 2}-f^{\alpha, 1, r}\right)(z) d z<\epsilon \quad \text { and } \quad \int\left|f^{\alpha, 1, r}(z+h)-f^{\alpha, 1, r}(z)\right| d z<\epsilon, \quad|h|<h_{0} .
$$


It can analogously be proven that for each $\kappa \in \mathbb{N},\left(f^{\alpha, \kappa}\right)_{\alpha \in] 0,1[}$ is translationally equicontinuous in $\alpha$. Let $\left(\alpha_{q}\right)_{q \in \mathbb{N}}$ be a sequence tending to zero. Take a subsequence in $\left(\alpha_{q}\right)_{q \in \mathbb{N}}$, still denoted by $\left(\alpha_{q}\right)_{q \in \mathbb{N}}$, such that $\left(f^{\alpha_{q}, 2}\right)_{q \in \mathbb{N}}$ converges in $L^{1}$ to some $f^{0,2}$ when $q \rightarrow+\infty$.

Continuing by induction gives a sequence $\left(f^{0, \kappa}\right)_{\kappa \in \mathbb{N}}$ satisfying

$$
\begin{aligned}
& f_{i}^{0,2 \kappa} \leq f_{i}^{0,2 \kappa+2} \leq F_{i}^{k} \leq f_{i}^{0,2 \kappa+3} \leq f_{i}^{0,2 \kappa+1}, \quad \kappa \in \mathbb{N}, \\
& v_{i} \cdot \nabla f_{i}^{0, \kappa+1}=G_{i}-\sum_{j, l, m=1}^{p} \Gamma_{i j}^{l m} \frac{f_{i}^{0, \kappa+1}}{1+\frac{f_{i}^{0, \kappa+1}}{k}} \frac{f_{j}^{0, \kappa}}{1+\frac{f_{j}^{0 \kappa}}{k}} \\
& f_{i}^{0, \kappa+1}\left(z_{i}^{+}(z)\right)=f_{b i}^{k}\left(z_{i}^{+}(z)\right) .
\end{aligned}
$$

Here, $G_{i}$ is the weak $L^{1}$ limit when $\alpha \rightarrow 0$ of the gain term

$$
\sum_{j, l, m=1}^{p} \Gamma_{i j}^{l m} \frac{F_{l}^{\alpha}}{1+\frac{F_{l}^{\alpha}}{k}} \frac{F_{m}^{\alpha} * \mu_{\alpha}}{1+\frac{F_{m}^{\alpha} * \mu_{\alpha}}{k}} .
$$

In particular, $\left(f_{i}^{0,2 \kappa}\right)_{\kappa \in \mathbb{N}}$ (resp. $\left.\left(f_{i}^{0,2 \kappa+1}\right)_{\kappa \in \mathbb{N}}\right)$ non decreasingly (resp. non increasingly) converges in $L^{1}$ to some $g_{i}$ (resp. $h_{i}$ ) when $\kappa \rightarrow+\infty$. The limits satisfy

$$
\begin{aligned}
& 0 \leq g_{i} \leq F_{i}^{k} \leq h_{i}, \\
& v_{i} \cdot \nabla h_{i}=G_{i}-\sum_{j, l, m=1}^{p} \Gamma_{i j}^{l m} \frac{h_{i}}{1+\frac{h_{i}}{k}} \frac{g_{j}}{1+\frac{g_{j}}{k}}, \\
& v_{i} \cdot \nabla g_{i}=G_{i}-\sum_{j, l, m=1}^{p} \Gamma_{i j}^{l m} \frac{g_{i}}{1+\frac{g_{i}}{k}} \frac{h_{j}}{1+\frac{h_{j}}{k}}, \\
& \left(h_{i}-g_{i}\right)\left(z_{i}^{+}(z)\right)=0 .
\end{aligned}
$$

Integrating and summing gives that

$$
\sum_{i=1}^{p} \int_{\partial \Omega_{i}^{-}}\left|v_{i} \cdot n(Z)\right|\left(h_{i}-g_{i}\right)(Z) d \sigma(Z)=0
$$

i.e. that $g_{i}=h_{i}$ also on $\partial \Omega_{i}^{-}$. Integrating the equation satisfied by $h_{i}-g_{i}$ over the part of $\Omega$ on one side of a line orthogonal to $n_{0}$, summing over $i$ and using (1.4) implies that $g=h$ on that line, hence in all of $\Omega$, and is equal to $F_{i}^{k}$. $\left(F^{\alpha_{q}}\right)_{q \in \mathbb{N}}$ converges to $F^{k}$ in $\left(L^{1}(\Omega)\right)^{p}$ when $q \rightarrow+\infty$. Indeed, given $\eta>0$, choose $\kappa_{0}$ big enough so that

$$
\left\|f_{i}^{0,2 \kappa_{0}+1}-f_{i}^{0,2 \kappa_{0}}\right\|_{L^{1}}<\eta \quad \text { and } \quad\left\|f_{i}^{0,2 \kappa_{0}}-F_{i}^{k}\right\|_{L^{1}}<\eta, \quad 1 \leq i \leq p,
$$

then $q_{0}$ big enough, so that

$$
\left\|f_{i}^{\alpha_{q}, 2 \kappa_{0}+1}-f_{i}^{0,2 \kappa_{0}+1}\right\|_{L^{1}} \leq \eta \quad \text { and } \quad\left\|f_{i}^{\alpha_{q}, 2 \kappa_{0}}-f_{i}^{0,2 \kappa_{0}}\right\|_{L^{1}} \leq \eta, \quad q \geq q_{0} .
$$

Then split $\left\|F_{i}^{\alpha_{q}}-F_{i}^{k}\right\|_{L^{1}}$ as follows,

$$
\begin{aligned}
& \left\|F_{i}^{\alpha_{q}}-F_{i}^{k}\right\|_{L^{1}} \\
& \leq\left\|F_{i}^{\alpha_{q}}-f_{i}^{\alpha, 2 \kappa_{0}}\right\|_{L^{1}}+\left\|f_{i}^{\alpha, 2 \kappa_{0}}-f_{i}^{0,2 \kappa_{0}}\right\|_{L^{1}}+\left\|f_{i}^{0,2 \kappa_{0}}-F_{i}^{k}\right\|_{L^{1}} \\
& \leq\left\|f_{i}^{\alpha, 2 \kappa_{0}+1}-f_{i}^{\alpha, 2 \kappa_{0}}\right\|_{L^{1}}+2 \eta \quad \text { by }(3.5) \\
& \leq\left\|f_{i}^{\alpha, 2 \kappa_{0}+1}-f_{i}^{0,2 \kappa_{0}+1}\right\|_{L^{1}}+\left\|f_{i}^{0,2 \kappa_{0}+1}-f_{i}^{0,2 \kappa_{0}}\right\|_{L^{1}}+\left\|f_{i}^{0,2 \kappa_{0}}-f_{i}^{\alpha, 2 \kappa_{0}}\right\|_{L^{1}}+2 \eta \\
& \leq 5 \eta, \quad q \geq q_{0} .
\end{aligned}
$$


Lemma 3.2 For any $k \in \mathbb{N}^{*}$, there is a nonnegative continuous solution $F^{k}$ to

$$
\begin{aligned}
& v_{i} \cdot \nabla F_{i}^{k}=Q_{i}^{+k}-F_{i}^{k} \nu_{i}^{k}, \\
& F_{i}^{k}\left(z_{i}^{+}(z)\right)=f_{b i}^{k}\left(z_{i}^{+}(z)\right), \quad 1 \leq i \leq p,
\end{aligned}
$$

where

$$
Q_{i}^{+k}=\sum_{j, l, m=1}^{p} \Gamma_{i j}^{l m} \frac{F_{l}^{k}}{1+\frac{F_{l}^{k}}{k}} \frac{F_{m}^{k}}{1+\frac{F_{m}^{k}}{k}}, \quad \nu_{i}^{k}=\sum_{j, l, m=1}^{p} \Gamma_{i j}^{l m} \frac{F_{j}^{k}}{\left(1+\frac{F_{i}^{k}}{k}\right)\left(1+\frac{F_{j}^{k}}{k}\right)} .
$$

Proof of Lemma 3.2.

Passing to the limit when $q \rightarrow+\infty$ in (2.19)-(2.20) written for $F^{\alpha_{q}}$, implies that $F^{k}$ is a solution in $\left(L_{+}^{1}(\Omega)\right)^{p}$ to $(3.9)-(3.10)$. It remains to prove its continuity. Using twice its exponential form and the continuity of $f_{b}^{k}$, it comes back to prove the continuity of

$$
\int_{0}^{s_{i}^{+}(z)} \int_{0}^{s_{j}^{+}\left(z_{i}^{+}(z)+s v_{i}\right)} G^{k}\left(z_{j}^{+}\left(z_{i}^{+}(z)+s v_{i}\right)+\sigma v_{j}\right) d \sigma d s, \quad i \neq j,
$$

for given measurable bounded functions $G^{k}$. The mapping

$$
(s, \sigma) \in\left[0, s_{i}^{+}(z)\right] \times\left[0, s_{j}^{+}\left(z_{i}^{+}(z)+s v_{i}\right)\right] \rightarrow Z=z_{j}^{+}\left(z_{i}^{+}(z)+s v_{i}\right)+\sigma v_{j},
$$

is a change of variables. Indeed, the strict convexity of $\Omega$ and the $C^{1}$ regularity of $\partial \Omega$ imply that $z \rightarrow z_{i}^{+}(z)$ is well-defined and $C^{1}$ for any $i \in\{1, \cdots, p\}$. Hence the map $(s, \sigma) \rightarrow Z$ is one to one and $C^{1}$. Its Jacobian equals one since $Z=Z_{i} v_{i}+Z_{j} v_{j}$, with $Z_{i}=s-s_{i}^{+}(z)$ linear in $s$ and independent of $\sigma$, and $Z_{j}=\sigma-s_{j}^{+}\left(z+\left(s-s_{i}^{+}(z)\right) v_{i}\right)$ linear in $\sigma$.

Using this change of variable leads to the continuity of the map defined in (3.11).

Lemma 3.3 Solutions $\left(F^{k}\right)_{k \in \mathbb{N}^{*}}$ to (3.9)-(3.10) have mass and entropy dissipation bounded from above uniformly with respect to $k$.

Proof of Lemma 3.3.

Choose an orthonormal basis $\left(e_{x}, e_{y}\right)$ of $\mathbb{R}^{2}$ so that neither the $x$-direction nor the $y$-direction is parallel to any of $v_{1}, \ldots, v_{p}$. Observe that integrating (3.9)-(3.10) over $\Omega$ and summing over $i$, shows that outflow of mass equals inflow. We shall first obtain uniformly in $k$, an upper bound for the energy

$$
\sum_{i=1}^{p} v_{i}^{2} \int_{\Omega} F_{i}^{k}(z) d z
$$

Recalling that the genericity condition (1.3) implies that all velocities are different from zero, the energy bound implies an upper estimate for the mass. Write $v_{i}=\xi_{i} e_{x}+\zeta_{i} e_{y}$. Multiply the equation for $F_{i}^{k}$ with $\xi_{i}$ and integrate over $\Omega_{a}=\Omega \cap\{(x, y) ; x \leq a\}$. Set

$$
S_{a}=\Omega \cap\{(x, y) ; x=a\} \quad \text { and } \quad \partial \Omega_{a}=\partial \Omega \cap \bar{\Omega}_{a} .
$$

From (3.9)-(3.10) follows

$$
\sum_{i=1}^{p} \xi_{i}^{2} \int_{S_{a}} F_{i}^{k}(a, y) d y=\sum_{i=1}^{p} \xi_{i} \int_{\partial \Omega_{a}}\left(v_{i} \cdot n(Z)\right) F_{i}^{k}(Z) d \sigma(Z) .
$$


For any $(x, y) \in \Omega$ let the line-segment through $(x, y)$ in the $x$-direction (resp. $y$-direction) intersect the boundary $\partial \Omega$ at $x^{-}(y)<x^{+}(y)$ (resp. $\left.y^{-}(x)<y^{+}(x)\right)$. Denote by

$$
x_{0}^{-}:=\min _{(x, y) \in \Omega}\left\{x^{-}(y)\right\}, \quad x_{0}^{+}:=\max _{(x, y) \in \Omega}\left\{x^{+}(y)\right\} .
$$

Integrating (3.13) on $a \in\left[x_{0}^{-}, x_{0}^{+}\right]$gives uniformly in $k$,

$$
\sum_{i=1}^{p} \xi_{i}^{2} \int_{\Omega} F_{i}^{k}(z) d z=\sum_{i=1}^{p} \xi_{i} \int_{x_{0}^{-}}^{x_{0}^{+}}\left(\int_{\partial \Omega_{a}}\left(v_{i} \cdot n(Z)\right) F_{i}^{k}(Z) d \sigma(Z)\right) d a \leq c_{b},
$$

where $c_{b}$ only depends on the given inflow. Analogously $\sum_{i=1}^{p} \zeta_{i}^{2} \int_{\Omega} F_{i}^{k}(z) d z \leq c_{b}$. The boundedness of energy and with it mass, follows.

The entropy dissipation estimate is proved as follows. Denote by $D^{k}$ the entropy production term for the approximation $F^{k}$,

$$
D^{k}=\sum_{i j l m} \Gamma_{i j}^{l m} \int_{\Omega}\left(\frac{F_{i}^{k}}{1+\frac{F_{i}^{k}}{k}} \frac{F_{j}^{k}}{1+\frac{F_{j}^{k}}{k}}-\frac{F_{l}^{k}}{1+\frac{F_{l}^{k}}{k}} \frac{F_{m}^{k}}{1+\frac{F_{m}^{k}}{k}}\right) \ln \frac{F_{i}^{k} F_{j}^{k}\left(1+\frac{F_{l}^{k}}{k}\right)\left(1+\frac{F_{m}^{k}}{k}\right)}{\left(1+\frac{F_{i}^{k}}{k}\right)\left(1+\frac{F_{j}^{k}}{k}\right) F_{l}^{k} F_{m}^{k}}(z) d z .
$$

Multiply (3.9) by $\ln \frac{F_{i}^{k}}{1+\frac{F_{i}^{k}}{k}}$, add the equations in $i$, and integrate the resulting equation on $\Omega$. It leads to

$$
\sum_{i=1}^{p} \int_{\partial \Omega_{i}^{-}}\left(F_{i}^{k} \ln F_{i}^{k}-k\left(1+\frac{F_{i}^{k}}{k}\right) \ln \left(1+\frac{F_{i}^{k}}{k}\right)\right)(Z)\left|v_{i} \cdot n(Z)\right| d \sigma(Z)+D^{k} \leq c_{b} .
$$

Moreover,

$$
k \int_{\partial \Omega_{i}^{-}} \ln \left(1+\frac{F_{i}^{k}}{k}\right)(Z)\left|v_{i} \cdot n(Z)\right| d \sigma(Z) \leq \int_{\partial \Omega_{i}^{-}} F_{i}^{k}(Z)\left|v_{i} \cdot n(Z)\right| d \sigma(Z) \leq c_{b} .
$$

Hence

$$
\sum_{i=1}^{p} \int_{\partial \Omega_{i}^{-}} F_{i}^{k} \ln \frac{F_{i}^{k}}{1+\frac{F_{i}^{k}}{k}}(Z)\left|v_{i} \cdot n(Z)\right| d \sigma(Z)+D^{k} \leq c_{b}
$$

The uniform entropy dissipation bound holds, since $x \rightarrow x \ln \frac{1+\frac{x}{k}}{x}$ is bounded from above on $] 0,+\infty[$.

The following lemma replaces an entropy control of $\left(F^{k}\right)_{k \in \mathbb{N}^{*}}$, under the condition (1.4).

Lemma 3.4 Assuming (1.4), it holds that

$$
\sum_{i=1}^{p} \int_{z \in \Omega ; F_{i}^{k}(z)<k} F_{i}^{k} \ln F_{i}^{k}(z) d z+\ln k \sum_{i=1}^{p} \int_{z \in \Omega ; F_{i}^{k}(z) \geq k} F_{i}^{k}(z) d z<c_{b}, \quad k \in \mathbb{N}^{*},
$$

where $c_{b}$ only depends on the given inflow. 


\section{Proof of Lemma 3.4.}

The entropy flow of $\left(F_{i}^{k}\right)$ is first controlled as follows. It holds that

$$
\begin{aligned}
& \int_{\partial \Omega_{i}^{-}} F_{i}^{k} \ln \left(1+\frac{F_{i}^{k}}{k}\right)(Z)\left|v_{i} \cdot n(Z)\right| d \sigma(Z) \\
& \leq \int_{\partial \Omega_{i}^{-}, F_{i}^{k} \leq k} F_{i}^{k} \ln \left(1+\frac{F_{i}^{k}}{k}\right)(Z)\left|v_{i} \cdot n(Z)\right| d \sigma(Z) \\
& +\int_{\partial \Omega_{i}^{-}, F_{i}^{k} \geq k} F_{i}^{k} \ln \left(1+\frac{F_{i}^{k}}{k}\right)(Z)\left|v_{i} \cdot n(Z)\right| d \sigma(Z) \\
& \leq \ln 2 \int_{\partial \Omega_{i}^{-}} F_{i}^{k}(Z)\left|v_{i} \cdot n(Z)\right| d \sigma(Z)+\int_{\partial \Omega_{i}^{-}, F_{i}^{k} \geq k} F_{i}^{k} \ln \frac{2 F_{i}^{k}}{k}(Z)\left|v_{i} \cdot n(Z)\right| d \sigma(Z) \\
& \leq c_{b}+\int_{\partial \Omega_{i}^{-}, F_{i}^{k} \geq k} F_{i}^{k} \ln F_{i}^{k}(Z)\left|v_{i} \cdot n(Z)\right| d \sigma(Z)-\ln \frac{k}{2} \int_{\partial \Omega_{i}^{-}, F_{i}^{k} \geq k} F_{i}^{k}(Z)\left|v_{i} \cdot n(Z)\right| d \sigma(Z) .
\end{aligned}
$$

Together with (3.15), this implies that

$$
\sum_{i=1}^{p} \int_{\partial \Omega_{i}^{-}, F_{i}^{k} \leq k} F_{i}^{k} \ln F_{i}^{k}(Z)\left|v_{i} \cdot n(Z)\right| d \sigma(Z)+\ln \frac{k}{2} \int_{\partial \Omega^{-}, F_{i}^{k} \geq k} F_{i}^{k}\left|v_{i} \cdot n(Z)\right| d \sigma(Z) \leq c_{b} .
$$

Set

$$
e_{x}:=n_{0}, \quad \Omega_{a}=\Omega \cap\{(x, y) ; x \leq a\}, \quad S_{a}=\Omega \cap\{(x, y) ; x=a\}, \quad \partial \Omega_{a}=\partial \Omega \cap \bar{\Omega}_{a} .
$$

Multiplying the equation for $F_{i}^{k}$ by $\ln \frac{F_{i}^{k}}{1+\frac{F_{i}^{k}}{k}}, 1 \leq i \leq p$, summing the resulting equations and integrating over $\Omega_{a}$, implies that

$$
\begin{aligned}
& \sum_{i=1}^{p} v_{i} \cdot n_{0} \int_{S_{a}}\left(F_{i}^{k} \ln F_{i}^{k}-k\left(1+\frac{F_{i}^{k}}{k}\right) \ln \left(1+\frac{F_{i}^{k}}{k}\right)\right)(a, y) d y \\
& \leq-D_{k}+\sum_{i=1}^{p} \int_{\partial \Omega_{a}}\left(F_{i}^{k} \ln F_{i}^{k}-k\left(1+\frac{F_{i}^{k}}{k}\right) \ln \left(1+\frac{F_{i}^{k}}{k}\right)\right)(Z)\left(v_{i} \cdot n(Z)\right) d \sigma(Z) \\
& \leq c_{b} .
\end{aligned}
$$

An integration on $\left[x_{0}^{-}, x_{0}^{+}\right]$defined in (3.14) implies that

$$
\sum_{i=1}^{p} v_{i} \cdot n_{0} \int_{\Omega}\left(F_{i}^{k} \ln F_{i}^{k}-k\left(1+\frac{F_{i}^{k}}{k}\right) \ln \left(1+\frac{F_{i}^{k}}{k}\right)\right)(z) d z \leq c_{b}
$$

Moreover,

$$
k \int_{\Omega} \ln \left(1+\frac{F_{i}^{k}}{k}\right)(z) d z \leq \int_{\Omega} F_{i}^{k}(z) d z \leq c_{b}, \quad 1 \leq i \leq p
$$


and

$$
\begin{aligned}
& \int_{\Omega} F_{i}^{k} \ln \left(1+\frac{F_{i}^{k}}{k}\right)(z) d z \\
& \leq \int_{z \in \Omega ; F_{i}^{k}(z) \leq k} F_{i}^{k} \ln \left(1+\frac{F_{i}^{k}}{k}\right)(z) d z+\int_{z \in \Omega ; F_{i}^{k}(z) \geq k} F_{i}^{k} \ln \left(1+\frac{F_{i}^{k}}{k}\right)(z) d z \\
& \leq \ln 2 \int_{\Omega} F_{i}^{k}(z) d z+\int_{z \in \Omega, F_{i}^{k}(z) \geq k} F_{i}^{k} \ln \frac{2 F_{i}^{k}}{k}(z) d z \\
& \leq c_{b}+\int_{z \in \partial \Omega ; F_{i}^{k}(z) \geq k} F_{i}^{k} \ln F_{i}^{k}(z) d z-\ln \frac{k}{2} \int_{z \in \Omega ; F_{i}^{k}(z) \geq k} F_{i}^{k}(z) d z .
\end{aligned}
$$

And so,

$$
\sum_{i=1}^{p} v_{i} \cdot n_{0}\left(\int_{z \in \Omega, F_{i}^{k}(z)<k} F_{i}^{k} \ln F_{i}^{k}(z) d z+\ln \frac{k}{2} \int_{z \in \Omega ; F_{i}^{k}(z) \geq k} F_{i}^{k}(z) d z\right)<c_{b} .
$$

The use of assumption (1.4) gives

$$
\int_{z \in \Omega, F_{i}^{k}(z)<k} F_{i}^{k} \ln F_{i}^{k}(z) d z+\ln \frac{k}{2} \int_{z \in \Omega ; F_{i}^{k}(z) \geq k} F_{i}^{k}(z) d z<c_{b}, \quad 1 \leq i \leq p, \quad k>2 .
$$

\section{The passage to the limit in the approximations.}

This section contains the proof of Theorem 1.1. The main part is a proof of strong $L^{1}$ compactness of $\left(F^{k}\right)_{k \in \mathbb{N}^{*}}$, based on two compactness lemmas for integrated collision frequency and gain term. Recall the exponential multiplier form for the approximations $\left(F^{k}\right)_{k \in \mathbb{N}^{*}}$,

$$
\begin{aligned}
F_{i}^{k}(z) & =f_{b i}^{k}\left(z_{i}^{+}(z)\right) e^{-\int_{0}^{s_{i}^{+}(z)} \nu_{i}^{k}\left(z_{i}^{+}(z)+s v_{i}\right) d s} \\
& +\int_{0}^{s_{i}^{+}(z)} Q_{i}^{+k}\left(z_{i}^{+}(z)+s v_{i}\right) e^{-\int_{s}^{s_{i}^{+}(z)} \nu_{i}^{k}\left(F^{k}\right)\left(z_{i}^{+}(z)+r v_{i}\right) d r} d s, \quad \text { a.a. } z \in \Omega, \quad 1 \leq i \leq p,
\end{aligned}
$$

where $\nu_{i}^{k}$ and $Q_{i}^{+k}$ are defined by

$$
\nu_{i}^{k}=\sum_{j l m} \Gamma_{i j}^{l m} \frac{F_{j}^{k}}{\left(1+\frac{F_{i}^{k}}{k}\right)\left(1+\frac{F_{j}^{k}}{k}\right)}, \quad Q_{i}^{+k}=\sum_{j l m} \Gamma_{i j}^{l m} \frac{F_{l}^{k}}{1+\frac{F_{l}^{k}}{k}} \frac{F_{m}^{k}}{1+\frac{F_{m}^{k}}{k}} .
$$

An $i$-characteristics is a segment of points $\left[Z-s_{i}^{+}(Z) v_{i}, Z\right]$, where $Z \in \partial \Omega_{i}^{-}$.

By the strict convexity of $\Omega$, there are for every $i \in\{1, \cdots p\}$ two points of $\partial \Omega$, denoted by $\tilde{Z}_{i}$ and $\bar{Z}_{i}$ such that

$$
z_{i}^{+}\left(\tilde{Z}_{i}\right)=z_{i}^{-}\left(\tilde{Z}_{i}\right) \quad \text { and } \quad z_{i}^{+}\left(\bar{Z}_{i}\right)=z_{i}^{-}\left(\bar{Z}_{i}\right)
$$

Denote by $\Omega_{i \epsilon}^{k 2}$ (resp. $\Omega_{i \epsilon}^{k 3}$ ) the set of points between $\tilde{Z}_{i}$ (resp. $\bar{Z}_{i}$ ) and the $i$-characteristics in $\Omega$ at distance $\epsilon$ from $\tilde{Z}_{i}$ (resp. $\bar{Z}_{i}$ ). Such subsets of $\Omega$ are introduced in order that all $i$-characteristics from $Z \in\left(\Omega_{i \epsilon}^{k 2} \cup \Omega_{i \epsilon}^{k 3}\right)^{c}$ are segments of length uniformly bounded from below in terms of $\epsilon$. 


\section{Lemma 4.1}

For $k \in \mathbb{N}^{*}, i \in\{1, \ldots, p\}$ and $\epsilon>0$, there is a subset $\Omega_{i \epsilon}^{k}$ of $i$-characteristics of $\Omega$ with measure smaller than $c_{b} \epsilon$, containing $\Omega_{i \epsilon}^{k 2}$ or $\Omega_{i \epsilon}^{k 3}$ defined above, and such that for any $z \in \Omega \backslash \Omega_{i \epsilon}^{k}$,

$$
F_{i}^{k}(z) \leq \frac{1}{\epsilon} \exp \left(\frac{1}{\epsilon}\right), \quad \int_{-s_{i}^{+}(z)}^{s_{i}^{-}(z)} \nu_{i}^{k}\left(z+s v_{i}\right) d s \leq \frac{1}{\epsilon} .
$$

Proof of Lemma 4.1.

It follows from the exponential form of $F_{i}^{k}$ that

$$
F_{i}^{k}(z) \leq F_{i}^{k}\left(z+s_{i}^{-}(z) v_{i}\right) e^{\int_{-s_{i}^{-}(z)}^{s_{i}^{-}(z)} \nu_{i}^{k}\left(z+r v_{i}\right) d r}, \quad z \in \Omega .
$$

The boundedness of the mass flow of $\left(F_{i}^{k}\right)_{k \in \mathbb{N}^{*}}$ across $\partial \Omega_{i}^{-}$is

$$
\int_{\partial \Omega_{i}^{-}}\left|v_{i} \cdot n(Z)\right| F_{i}^{k}(Z) d \sigma(Z) \leq c_{b} .
$$

Consequently, the measure of the set $\left\{Z \in \partial \Omega_{i}^{-} ; F_{i}^{k}(Z)>\frac{1}{\epsilon}\right\}$ is smaller than $c_{b} \epsilon$.

The boundedness of the mass of $\left(F_{j}^{k}\right)_{k \in \mathbb{N}^{*}, 1 \leq j \leq p}$ can be written

$$
\int_{\Omega} F_{j}^{k}(z) d z=\int_{\partial \Omega_{i}^{-}}\left|v_{i} \cdot n(Z)\right|\left(\int_{-s_{i}^{+}(Z)}^{0} F_{j}^{k}\left(Z+r v_{i}\right) d r\right) d \sigma(Z) \leq c_{b} .
$$

Hence the measure of the set

$$
\left\{Z \in \partial \Omega_{i}^{-} ; \int_{-s_{i}^{+}(Z)}^{0} F_{j}^{k}\left(Z+r v_{i}\right) d r>\frac{p^{2} \Gamma}{\epsilon}\right\},
$$

where $\Gamma=\max _{i, j, k, l} \Gamma_{i j}^{l m}$, is smaller than $c_{b} \epsilon$. Hence the measure of the set of $Z \in \partial \Omega_{i}^{-}$outside of which $F_{i}^{k}(Z) \leq \frac{1}{\epsilon}$ and $\int_{-s_{i}^{-}(Z)}^{0} F_{j}^{k}\left(Z+r v_{i}\right) d r \leq \frac{p^{2} \Gamma}{\epsilon}$, is bounded by $c_{b} \epsilon$. Together with (4.3), this implies that the measure of the complement of the set of $Z \in \partial \Omega_{i}^{-}$, such that

$$
F_{i}^{k}(z) \leq \frac{1}{\epsilon} \exp \left(\frac{1}{\epsilon}\right) \quad \text { and } \quad \int_{-s_{i}^{+}(z)}^{s_{i}^{-}(z)} \nu_{i}^{k}\left(z+r v_{i}\right) d r \leq \frac{1}{\epsilon}
$$

for $z=Z-s v_{i}, \quad 0 \leq s \leq s_{i}^{+}(Z)$, is bounded by $2 c_{b} \epsilon$. With it $2 c_{b} \epsilon$ is a bound for the measure of the complement, denoted by $\Omega_{i \epsilon}^{k 1}$, of the set of $i$-characteristics in $\Omega$ such that for all points $z$ on the $i$-characteristics,

$$
F_{i}^{k}(z) \leq \frac{1}{\epsilon} e^{\frac{1}{\epsilon}} \quad \text { and } \quad \int_{-s_{i}^{+}(z)}^{s_{i}^{-}(z)} \nu_{i}^{k}\left(z+r v_{i}\right) d r \leq \frac{1}{\epsilon}
$$

The sets of points $\Omega_{i \epsilon}^{k 2}$ and $\Omega_{i \epsilon}^{k 3}$ have measure of magnitude $\epsilon$, and are also included in $\Omega_{i \epsilon}^{k}$,

$$
\Omega_{i \epsilon}^{k}=\cup_{p=1}^{3} \Omega_{i \epsilon}^{k p} .
$$

This ends the proof of the lemma.

Given $i \in\{1, \ldots, p\}$ and $\epsilon$ in Lemma 4.1, let $\chi_{i \epsilon}^{k}$ denote the characteristic function of the complement of $\Omega_{i \epsilon}^{k}$. The following lemma proves the compactness of the $k$-sequence of integrated collision frequencies. 


\section{Lemma 4.2}

The sequences

$$
\left(\int_{0}^{s_{i}^{+}(z)} \nu_{i}^{k}\left(z_{i}^{+}(z)+s v_{i}\right) d s\right)_{k \in \mathbb{N}^{*}}, \quad 1 \leq i \leq p
$$

are strongly compact in $L^{1}(\Omega)$.

Proof of Lemma 4.2.

Let $1 \leq i \leq p$. The uniform bound for the mass of $\left(F^{k}\right)$ proven in Lemma 3.3, implies that

$$
\int_{\Omega}\left(\int_{0}^{s_{i}^{+}(z)} \nu_{i}^{k}\left(z_{i}^{+}(z)+s v_{i}\right) d s\right) d z
$$

is uniformly bounded with respect to $k$. By the Kolmogorov-Riesz theorem ([10], [11]), the compactness will follow from the translational equi-continuity in $L^{1}(\Omega)$. The translational equi-continuity in the $v_{i}$-direction of $\left(\int_{0}^{s_{i}^{+}(z)} \nu_{i}^{k}\left(z_{i}^{+}(z)+s v_{i}\right) d s\right)_{k \in \mathbb{N}^{*}}$ follows from the previous uniform bound on $\int_{\Omega}\left(\int_{0}^{s_{i}^{+}(z)} \nu_{i}^{k}\left(z_{i}^{+}(z)+s v_{i}\right) d s\right) d z$. Let us prove the translational equi-continuity in the $v_{j}$-direction of each of its terms,

$$
\Gamma_{i j}^{l m} \int_{0}^{s_{i}^{+}(z)} \frac{F_{j}^{k}}{\left(1+\frac{F_{i}^{k}}{k}\right)\left(1+\frac{F_{j}^{k}}{k}\right)}\left(z_{i}^{+}(z)+s v_{i}\right) d s .
$$

It follows from the weak $L^{1}$ - compactness of $\left(F^{k}\right)_{k \in \mathbb{N}^{*}}$ that

$$
\int_{\Omega}\left(\int_{0}^{s_{i}^{+}(z)}\left(\left(1-\chi_{j \epsilon}^{k}\right) \frac{F_{j}^{k}}{\left(1+\frac{F_{i}^{k}}{k}\right)\left(1+\frac{F_{j}^{k}}{k}\right)}\left(z_{i}^{+}(z)+s v_{i}\right) d s\right) d z\right.
$$

can be made arbitrarily small for $\epsilon$ small enough. Consider the remaining term in which $\chi_{j \epsilon}^{k} F_{j}^{k}$ is bounded by $\frac{1}{\epsilon} \exp \left(\frac{1}{\epsilon}\right)$. Noticing that the translational difference of $\frac{F_{j}^{k}}{k}$ tends to zero, when $k$ tends to infinity, there remains to study the translational difference in the $v_{j}$-direction of

$$
\int_{0}^{s_{i}^{+}(z)}\left(\chi_{j \epsilon}^{k} F_{j}^{k}\right)\left(z_{i}^{+}(z)+s v_{i}\right) d s .
$$

Write $F_{j}^{k}\left(z_{i}^{+}(z)+s v_{i}\right)$ in exponential multiplier form,

$$
\int_{0}^{s_{i}^{+}(z)}\left(\chi_{j \epsilon}^{k} F_{j}^{k}\right)\left(z_{i}^{+}(z)+s v_{i}\right) d s=A_{i, j}^{k}(z)+B_{i, j}^{k}(z),
$$

where

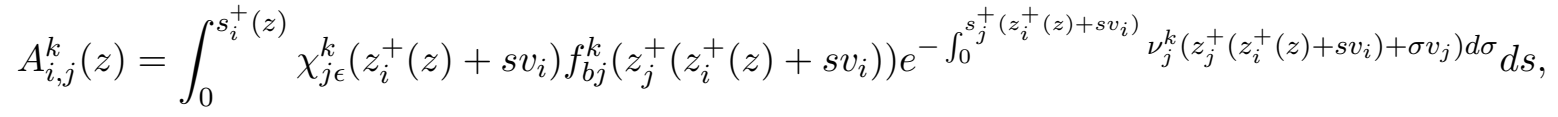

$$
\begin{aligned}
& B_{i, j}^{k}(z)=\int_{0}^{s_{i}^{+}(z)} \chi_{j \epsilon}^{k}\left(z_{i}^{+}(z)+s v_{i}\right) \int_{0}^{s_{j}^{+}\left(z_{i}^{+}(z)+s v_{i}\right)} Q_{j}^{+k}\left(z_{j}^{+}\left(z_{i}^{+}(z)+s v_{i}\right)+\sigma v_{j}\right)
\end{aligned}
$$

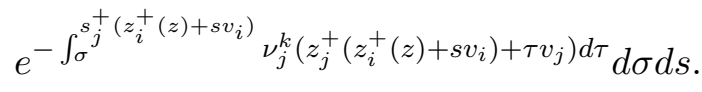


In order to prove the translational equicontinuity of $\left(A_{i, j}^{k}\right)$, it is sufficient to prove the translational equicontinuity of

$$
\left(\int_{0}^{s_{i}^{+}(z)} \chi_{j \epsilon}^{k}\left(z_{i}^{+}(z)+s v_{i}\right) \int_{0}^{s_{j}^{+}\left(z_{i}^{+}(z)+s v_{i}\right)} \nu_{j}^{k}\left(z_{j}^{+}\left(z_{i}^{+}(z)+s v_{i}\right)+\sigma v_{j}\right) d \sigma d s\right)_{k \in \mathbb{N}^{*}},
$$

by the $L_{v_{i} \cdot n(Z)}^{1}\left(\partial \Omega^{+}\right)$compactness of $\left(f_{b j}^{k}\left(z_{j}^{+}\left(z_{i}^{+}(z)+s v_{i}\right)\right)\right)_{k \in \mathbb{N}^{*}}$. It is so since, by the change of variables (3.12), each of its terms is a linear combination of

$$
\int_{a_{i, j}(z)} \frac{F_{l}^{k}}{\left(1+\frac{F_{j}^{k}}{k}\right)\left(1+\frac{F_{l}^{k}}{k}\right)}(Z) d Z, \quad 1 \leq l \leq p,
$$

with domains $a_{i, j}(z) \subset \Omega$, continuously depending on $z \in \Omega$, and such that

$$
\left|a_{i, j}(z) \backslash a_{i, j}(z+h)\right| \leq c h, \quad z \in \Omega
$$

uniformly with respect to $z$.

The integral where $F_{l}^{k}>\Lambda$, tends to zero when $\Lambda \rightarrow \infty$. If $F_{l}^{k}>\Lambda$ at one but not the other of the two terms in the translation difference, then moving the evaluation points closer, by continuity the larger value of $F_{l}^{k}$ can be changed to $\Lambda$. And so we can assume $F_{l}^{k}$ bounded at both evaluation points in the translation difference. It follows that $\left(A_{i, j}^{k}\right)_{k \in \mathbb{N}^{*}}$ is translationally equi-continuous. $B_{i, j}^{k}$ is a sum of

$$
\begin{aligned}
& \Gamma_{j j^{\prime}}^{l m} \int_{0}^{s_{i}^{+}(z)} \chi_{j \epsilon}^{k}\left(z_{i}^{+}(z)+s v_{i}\right) \int_{0}^{s_{j}^{+}\left(z_{i}^{+}(z)+s v_{i}\right)} \frac{F_{l}^{k} F_{m}^{k}}{\left(1+\frac{F_{l}^{k}}{k}\right)\left(1+\frac{F_{m}^{k}}{k}\right)}\left(z_{j}^{+}\left(z_{i}^{+}(z)+s v_{i}\right)+\sigma v_{j}\right)
\end{aligned}
$$

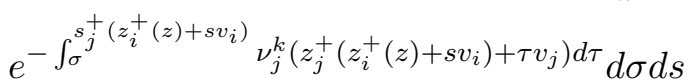

terms. Consider each one of these terms and split it into $\Gamma_{j j^{\prime}}^{l m}\left(C_{1}^{k}+C_{2}^{k}+C_{3}^{k}\right)$, where, for real numbers $J_{1}$ and $J_{2}$ to be fixed later,

$$
\begin{aligned}
& C_{1}^{k}(z)=\int_{0}^{s_{i}^{+}(z)} \chi_{j \epsilon}^{k}\left(z_{i}^{+}(z)+s v_{i}\right) \int_{0}^{s_{j}^{+}\left(z_{i}^{+}(z)+s v_{i}\right)} \frac{F_{l}^{k} F_{m}^{k}}{\left(1+\frac{F_{l}^{k}}{k}\right)\left(1+\frac{F_{m}^{k}}{k}\right)}\left(z_{j}^{+}\left(z_{i}^{+}(z)+s v_{i}\right)+\sigma v_{j}\right) \\
& 1 \frac{F_{l}^{k} F_{m}^{k}}{\left(1+\frac{F_{l}^{k}}{k}\right)\left(1+\frac{F_{m}^{k}}{k}\right)}\left(z_{j}^{+}\left(z_{i}^{+}(z)+s v_{i}\right)+\sigma v_{j}\right)>J_{1} \frac{F_{j}^{k} F_{j^{\prime}}^{k}}{\left(1+\frac{F_{j}^{k}}{k}\right)\left(1+\frac{F_{j^{\prime}}^{k}}{k}\right)}\left(z_{j}^{+}\left(z_{i}^{+}(z)+s v_{i}\right)+\sigma v_{j}\right)
\end{aligned}
$$

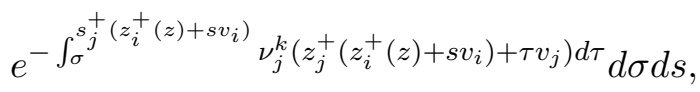

$$
\begin{aligned}
& C_{2}^{k}(z)=\int_{0}^{s_{i}^{+}(z)} \chi_{j \epsilon}^{k}\left(z_{i}^{+}(z)+s v_{i}\right) \int_{0}^{s_{j}^{+}\left(z_{i}^{+}(z)+s v_{i}\right)} \frac{F_{l}^{k} F_{m}^{k}}{\left(1+\frac{F_{l}^{k}}{k}\right)\left(1+\frac{F_{m}^{k}}{k}\right)}\left(z_{j}^{+}\left(z_{i}^{+}(z)+s v_{i}\right)+\sigma v_{j}\right) \\
& 1 \frac{F_{l}^{k} F_{m}^{k}}{\left(1+\frac{F_{l}^{k}}{k}\right)\left(1+\frac{F_{m}^{k}}{k}\right)}\left(z_{j}^{+}\left(z_{i}^{+}(z)+s v_{i}\right)+\sigma v_{j}\right)<J_{1} \frac{F_{j}^{k} F_{j^{\prime}}^{k}}{\left(1+\frac{F_{j}^{k}}{k}\right)\left(1+\frac{F_{j^{\prime}}^{k}}{k}\right)}\left(z_{j}^{+}\left(z_{i}^{+}(z)+s v_{i}\right)+\sigma v_{j}\right), F_{j^{\prime}}^{k}\left(z_{j}^{+}\left(z_{i}^{+}(z)+s v_{i}\right)+\sigma v_{j}\right)>J_{2}
\end{aligned}
$$

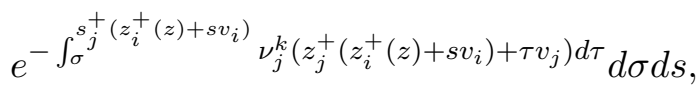




$$
\begin{aligned}
& C_{3}^{k}(z)=\int_{0}^{s_{i}^{+}(z)} \chi_{j \epsilon}^{k}\left(z_{i}^{+}(z)+s v_{i}\right) \int_{0}^{s_{j}\left(z_{i}^{+}(z)+s v_{i}\right)} \frac{F_{l}^{k} F_{m}^{k}}{\left(1+\frac{F_{l}^{k}}{k}\right)\left(1+\frac{F_{m}^{k}}{k}\right)}\left(z_{j}^{+}\left(z_{i}^{+}(z)+s v_{i}\right)+\sigma v_{j}\right) \\
& 1 \frac{F_{l}^{k} F_{m}^{k}}{\left(1+\frac{F_{l}^{k}}{k}\right)\left(1+\frac{F_{m}^{k}}{k}\right)}\left(z_{j}^{+}\left(z_{i}^{+}(z)+s v_{i}\right)+\sigma v_{j}\right)<J_{1} \frac{F_{j}^{k} F_{j^{\prime}}^{k}}{\left(1+\frac{F_{j}^{k}}{k}\right)\left(1+\frac{F_{j^{\prime}}^{k}}{k}\right)}\left(z_{j}^{+}\left(z_{i}^{+}(z)+s v_{i}\right)+\sigma v_{j}\right), F_{j^{\prime}}^{k}\left(z_{j}^{+}\left(z_{i}^{+}(z)+s v_{i}\right)+\sigma v_{j}\right)<J_{2}
\end{aligned}
$$

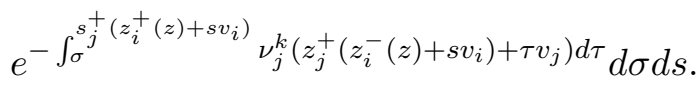

Using the uniform boundedness of the entropy production terms $\left(D^{k}\right)_{k \in \mathbb{N}^{*}}$ and choosing $J_{1}$ large enough, $C_{1}^{k}$ can be made arbitrarily small, uniformly with respect to $k$. For such a $J_{1}$, notice that

$$
\begin{gathered}
C_{2}^{k} \leq \frac{J_{1} e^{\frac{1}{\epsilon}}}{\epsilon} \int_{0}^{s_{i}^{+}(z)} \chi_{j \epsilon}^{k}\left(z_{i}^{+}(z)+s v_{i}\right) \int_{0}^{s_{j}^{+}\left(z_{i}^{+}(z)+s v_{i}\right)} \frac{F_{j^{\prime}}^{k}}{1+\frac{F_{j^{\prime}}^{k}}{k}}\left(z_{j}^{+}\left(z_{i}^{+}(z)+s v_{i}\right)+\sigma v_{j}\right) \\
\frac{1}{\frac{F_{l}^{k} F_{m}^{k}}{\left(1+\frac{F_{l}^{k}}{k}\right)\left(1+\frac{F_{m}^{k}}{k}\right)}}\left(z_{j}^{+}\left(z_{j}^{+}\left(z_{i}^{+}(z)+s v_{i}\right)+\sigma v_{j}\right)<J_{1} \frac{F_{j}^{k} F_{j^{\prime}}^{k}}{\left(1+\frac{F_{j}^{k}}{k}\right)\left(1+\frac{F_{j^{\prime}}^{k}}{k}\right)}\left(z_{j}^{+}\left(z_{i}^{+}(z)+s v_{i}\right)+\sigma v_{j}\right), F_{j^{\prime}}^{k}\left(z_{j}^{+}\left(z_{i}^{+}(z)+s v_{i}\right)+\sigma v_{j}\right)>J_{2}\right. \\
e^{-\int_{\sigma}^{s_{j}^{+}\left(z_{i}^{+}(z)+s v_{i}\right)} \Gamma_{j j^{\prime}}^{l m} \frac{F_{j^{\prime}}^{k}}{1+\frac{F_{j^{\prime}}^{k}}{k}}\left(z_{j}^{+}\left(z_{i}^{+}(z)+s v_{i}\right)+\tau v_{j}\right) d \tau} d \sigma d s .
\end{gathered}
$$

By the continuity of $F^{k}$, the integral with respect to $\sigma$ is a sum of integrals over disjoint intervals where $F_{j^{\prime}}^{k}>J_{2}$. The total integral is bounded by an integral of $F_{j^{\prime}}^{k}$ over a set where $F_{j^{\prime}}^{k}>J_{2}$. Using the entropy control, $\int_{F_{j^{\prime}}^{k}>J_{2}} F_{j^{\prime}}^{k} \rightarrow 0$ when $J_{2} \rightarrow \infty$. And so choosing $J_{2}$ large enough, $C_{2}^{k}$ can be made arbitrarily small, uniformly with respect to $k$.

It follows from the boundedness of $\left(F_{l}^{k} F_{m}^{k}\right)$ on its domain of integration in $C_{3}^{k}$, that the closing argument in the proof of translational equi-continuity for $\left(A_{i, j}^{k}\right)$ above, can be used to conclude that $\left(C_{3}^{k}\right)_{k \in \mathbb{N}^{*}}$ and with it $\left(B_{i, j}^{k}\right)$ are translationally equi-continuous. This ends the proof of the lemma.

For any $i \in\{1, \cdots, p\}$, the following lemma proves the compactness of the integrated gain terms times $\chi_{i \epsilon}^{k}$ in the exponential multiplier form of the $\left(F_{i}^{k}\right)$-sequence, again by the Kolmogorov-Riesz theorem.

\section{Lemma 4.3}

Take $\epsilon>0$. The sequences

$$
\left(\chi_{i \epsilon}^{k}(z) \int_{0}^{s_{i}^{+}(z)} \frac{F_{l}^{k} F_{m}^{k}}{\left(1+\frac{F_{l}^{k}}{k}\right)\left(1+\frac{F_{m}^{k}}{k}\right)}\left(z_{i}^{+}(z)+s v_{i}\right) e^{-\int_{s}^{s_{i}^{+}(z)} \nu_{i}^{k}\left(z_{i}^{+}(z)+r v_{i}\right) d r} d s\right)_{k \in \mathbb{N}^{*}}, i=1, \ldots, p,
$$

are strongly compact in $L^{1}(\Omega)$.

Proof of Lemma 4.3.

The sequence $\left(F_{i}^{k}\right)_{k \in \mathbb{N}^{*}}$ being uniformly bounded in $L^{1}$, the same holds for (4.4). For proving its uniform $L^{1}$ equi-continuity, split the domain of integration in $(z, s) \in \Omega \times\left[0, s_{i}^{+}(z)\right]$ into the sets 
where

$$
\begin{aligned}
& \frac{F_{l}^{k} F_{m}^{k}}{\left(1+\frac{F_{l}^{k}}{k}\right)\left(1+\frac{F_{m}^{k}}{k}\right)}\left(z_{i}^{+}(z)+s v_{i}\right)>J_{1} \frac{F_{i}^{k} F_{j}^{k}}{\left(1+\frac{F_{i}^{k}}{k}\right)\left(1+\frac{F_{j}^{k}}{k}\right)}\left(z_{i}^{+}(z)+s v_{i}\right), \\
& \left(\operatorname{resp.} \frac{F_{l}^{k} F_{m}^{k}}{\left(1+\frac{F_{l}^{k}}{k}\right)\left(1+\frac{F_{m}^{k}}{k}\right)}\left(z_{i}^{+}(z)+s v_{i}\right)<J_{1} \frac{F_{i}^{k} F_{j}^{k}}{\left(1+\frac{F_{i}^{k}}{k}\right)\left(1+\frac{F_{j}^{k}}{k}\right)}\left(z_{i}^{+}(z)+s v_{i}\right)\right. \\
& \text { and } \left.F_{j}^{k}\left(z_{i}^{+}(z)+s v_{i}\right)>J_{2}\right),
\end{aligned}
$$

where the integrals are arbitrarily small for $J_{1}$ (resp. $J_{2}$ ) large enough, and the remaining domain,

$$
\begin{aligned}
X:= & \left\{(z, s) \in \Omega \times\left[0, s_{i}^{+}(z)\right] ; \frac{F_{l}^{k} F_{m}^{k}}{\left(1+\frac{F_{l}^{k}}{k}\right)\left(1+\frac{F_{m}^{k}}{k}\right)}\left(z_{i}^{+}(z)+s v_{i}\right)<J_{1} \frac{F_{i}^{k} F_{j}^{k}}{\left(1+\frac{F_{i}^{k}}{k}\right)\left(1+\frac{F_{j}^{k}}{k}\right)}\left(z_{i}^{+}(z)+s v_{i}\right)\right. \\
& \text { and } \left.F_{j}^{k}\left(z_{i}^{+}(z)+s v_{i}\right)<J_{2}\right\},
\end{aligned}
$$

where $\left(F_{l}^{k} F_{m}^{k}\right)$ is bounded uniformly with respect to $k$. Let us prove the $L^{1}$ uniform equi-continuity of

$$
\left(\chi_{i \epsilon}^{k}(z) \int_{0}^{s_{i}^{+}(z)} \frac{F_{l}^{k} F_{m}^{k}}{\left(1+\frac{F_{l}^{k}}{k}\right)\left(1+\frac{F_{m}^{k}}{k}\right)}\left(z_{i}^{+}(z)+s v_{i}\right) d s\right)_{k \in \mathbb{N}^{*}}
$$

on this domain. We can also restrict to a domain where both $F_{l}^{k}\left(z_{i}^{+}(z)+s v_{i}\right)$ and $F_{m}^{k}\left(z_{i}^{+}(z)+s v_{i}\right)$ are bounded, since

$$
\begin{aligned}
& \int_{(z, s) \in X ; s \in\left[0, s_{i}^{+}(z)\right], F_{l}^{k}\left(z_{i}^{+}(z)+s v_{i}\right) \geq \Lambda} \chi_{i \epsilon}^{k}(z) \frac{F_{l}^{k} F_{m}^{k}}{\left(1+\frac{F_{l}^{k}}{k}\right)\left(1+\frac{F_{m}^{k}}{k}\right)}\left(z_{i}^{+}(z)+s v_{i}\right) d s d z \\
& \leq \frac{J_{1} J_{2} e^{\frac{1}{\epsilon}}}{\epsilon}\left|\left\{(z, s) \in X ; s \in\left[0, s_{i}^{+}(z)\right], F_{l}^{k}\left(z_{i}^{+}(z)+s v_{i}\right) \geq \Lambda\right\}\right|
\end{aligned}
$$

and the measure of the set where $F_{l}^{k}>\Lambda$ tends to zero when $\Lambda \rightarrow+\infty$. And so, we have reduced the problem to proving the $L^{1}$ uniform equi-continuity of

$$
\left(\int_{0}^{s_{i}^{+}(z)} 1_{F_{l}^{k}\left(z_{i}^{+}(z)+s v_{i}\right)<\Lambda} \frac{F_{l}^{k}}{1+\frac{F_{l}^{k}}{k}}\left(z_{i}^{+}(z)+s v_{i}\right) d s\right)_{k \in \mathbb{N}^{*}},
$$

which follows from the proof of Lemma 4.2.

\section{Lemma 4.4}

Up to a subsequence $\left(F^{k}\right)_{k \in \mathbb{N}^{*}}$ strongly converges in $L^{1}(\Omega)$.

Proof of Lemma 4.4.

Let $F$ be a weak $L^{1}$ limit of a subsequence of $\left(F^{k}\right)_{k \in \mathbb{N}^{*}}$. For every $\epsilon>0$, the sequence $\left(\chi_{i \epsilon}^{k} F_{i}^{k}\right)_{k \in \mathbb{N}^{*}}$ is compact in $L^{1}(\Omega)$ by Lemmas 4.2-4.3. For a converging subsequence of $\left(\chi_{i \epsilon}^{k} F^{k}\right)_{k \in \mathbb{N}^{*}}$, the limit depends on $\epsilon$. Choose a decreasing sequence $\left(\epsilon_{q}\right)$ with $\lim _{q \rightarrow \infty} \epsilon_{q}=0$, and a diagonal subsequence in $k$ with $\chi_{i \epsilon_{q}}^{k}$ converging in $k$ for all $q$, and increasing with $q$. Split $F^{k}-F$ into

$$
\chi_{i \epsilon_{q}}^{k}\left(F_{i}^{k}-F_{i}\right)+\left(1-\chi_{i \epsilon_{q}}^{k}\right) F_{i}^{k}-\left(1-\chi_{i \epsilon_{q}}^{k}\right) F_{i}, \quad 1 \leq i \leq p .
$$


Using that $\int_{\Omega_{i \epsilon_{q}}^{k}} F_{i}^{k}$ and $\int_{\Omega_{i \epsilon_{q}}^{k}} F_{i}$ are arbitrarily small for $\epsilon_{q}$ small enough, leads to the result.

\section{Lemma 4.5}

Under the assumptions of Theorem 1.1, F is a nonnegative renormalized solution of the discrete velocity coplanar Boltzmann boundary value problem (1.6)-(1.7).

Proof of Lemma 4.5.

Start from a renormalized formulation for $\chi_{i \epsilon}^{k} F_{i}^{k}$,

$$
\begin{aligned}
& -\int_{\partial \Omega^{-}} \varphi_{i} \chi_{i \epsilon}^{k} \ln \left(1+F_{i}^{k}\right)(Z) v_{i} \cdot n(Z) d \sigma(Z)-\int_{\partial \Omega^{+}} \varphi_{i} \chi_{i \epsilon}^{k} \ln \left(1+f_{b i}^{k}\right)(Z) v_{i} \cdot n(Z) d \sigma(Z) \\
& -\int_{\Omega} \chi_{i \epsilon}^{k} \ln \left(1+F_{i}^{k}\right) v_{i} \cdot \nabla \varphi_{i}(z) d z \\
& =\int_{\Omega} \frac{\varphi_{i} \chi_{i \epsilon}^{k}}{1+F_{i}^{k}} \sum_{j, l, m=1}^{p} \Gamma_{i j}^{l m}\left(\frac{F_{l}^{k}}{1+\frac{F_{l}^{k}}{k}} \frac{F_{m}^{k}}{1+\frac{F_{m}^{k}}{k}}-\frac{F_{i}^{k}}{1+\frac{F_{i}^{k}}{k}} \frac{F_{j}^{k}}{1+\frac{F_{j}^{k}}{k}}\right) d z,
\end{aligned}
$$

for test functions $\varphi \in\left(C^{1}(\Omega)\right)^{p}$. Use the strong $L^{1}$ convergence given by Lemma 4.4 for the sequence $\left(F^{k}\right)_{k \in \mathbb{N}^{*}}$, to pass to the limit in the left hand side of (4.5) when $k \rightarrow+\infty$. This gives in the limit for the left hand side

$$
\begin{aligned}
& -\int_{\partial \Omega^{-}} \varphi_{i} \ln \left(1+F_{i}\right)(Z) v_{i} \cdot n(Z) d \sigma(Z)-\int_{\partial \Omega^{+}} \varphi_{i} \ln \left(1+f_{b i}\right)(Z) v_{i} \cdot n(Z) d \sigma(Z) \\
& -\int_{\Omega} \ln \left(1+F_{i}\right) v_{i} \nabla \varphi_{i}(z) d z .
\end{aligned}
$$

For the passage to the limit when $k \rightarrow+\infty$ in the right hand side of (4.5), given $\eta>0$ there is a subset $A_{\eta}$ of $\Omega$ with $\left|A_{\eta}^{c}\right|<\eta$, such that up to a subsequence, $\left(F_{k}\right)$ uniformly converges to $F$ on $A_{\eta}$ and $F \in L^{\infty}\left(A_{\eta}\right)$. Passing to the limit when $k \rightarrow+\infty$ on $A_{\eta}$ is straightforward. Moreover,

$$
\lim _{\eta \rightarrow 0} \int_{A_{\eta}^{c}} \frac{\varphi_{i}}{1+F_{i}} Q_{i}^{-}(F)(z) d z=0 \text { and } \lim _{\eta \rightarrow 0} \int_{A_{\eta}^{c}} \varphi_{i} \chi_{i \epsilon}^{k} F_{i}^{k} \nu_{i}^{k}(z) d z=0,
$$

uniformly with respect to $k$, since

$$
\frac{F_{i}}{1+F_{i}} \leq 1, \quad \frac{F_{i}^{k}}{\left(1+F_{i}^{k}\right)\left(1+\frac{F_{i}^{k}}{k}\right)\left(1+\frac{F_{j}^{k}}{k}\right)} \leq 1, \quad \text { and } \quad \lim _{\eta \rightarrow 0} \int_{A_{\eta}^{c}} F_{j}^{k}=0
$$

uniformly with respect to $k$. The passage to the limit in the loss term follows.

The passage to the limit in the gain term can be done as follows. The uniform boundedness of the entropy production term of $\left(F^{k}\right)$ given by (3.15) in Lemma 3.3, implies that for any $\gamma>1$,

$$
\int_{A_{\eta}^{c}}\left|\varphi_{i}\right| \frac{\chi_{i}^{k}}{1+F_{i}^{k}} \sum_{j, l, m=1}^{p} \Gamma_{i j}^{l m} \frac{F_{l}^{k}}{1+\frac{F_{l}^{k}}{k}} \frac{F_{m}^{k}}{1+\frac{F_{m}^{k}}{k}}(z) d z \leq \frac{c}{\ln \gamma}+c \gamma \int_{A_{\eta}^{c}} F_{i}^{k} \nu_{i}^{k}(z) d z
$$

Take first $\gamma$ large, then $\eta$ small. It follows that the right hand side of (4.5) converges to

$$
\int_{\Omega} \varphi_{i} \frac{Q_{i}^{+}(F)}{1+F_{i}}(z) d z-\int_{\Omega} \varphi_{i} \frac{Q_{i}^{-}(F)}{1+F_{i}}(z) d z,
$$


when $k \rightarrow+\infty$. Consequently, $F$ satisfies (1.6)-(1.7) in renormalized form.

Remark 4.1 Strong $L^{1}$ compactness and convergence to a renormalized solution of the discrete velocity coplanar Boltzmann boundary value problem (1.6)-(1.7), as obtained in Section 4, would also hold without Assumption 1.4, for a sequence of approximations $\left(F^{k}\right)_{k \in \mathbb{N}}$ weakly compact in $L^{1}$. This will be the frame of a following paper.

\section{References}

[1] L. Arkeryd, A. Nouri, On the stationary Povzner equation in $\mathbb{R}^{n}$, J. Math. Kyoto Univ. 39 (1) (1999), 115-153.

[2] L. Arkeryd, A. Nouri, Stationary solutions to the two-dimensional Broadwell model, arXiv 2019.

[3] A. Bobylev, Exact solutions of discrete kinetic models and stationary problems for the plane Broadwell model, Math. Methods Appl. Sci. (4) 19 (1996), 825-845.

[4] A. Bobylev, A. Palczewski, J. Schneider, A consistency result for a discrete-velocity model of the Boltzmann equation, SIAM J. Numer. Anal. 34 (5) (1997), 1865-1883.

[5] C. Cercignani, Sur des critères d'existence globale en théorie cinétique discrète, C. R. Acad. Sc. Paris, 301 (1985), 89-92.

[6] C. Cercignani, R. Illner, M. Shinbrot, A boundary value problem for the 2-dimensional Broadwell model, Commun. Math. Phys. 114 (1988), 687-698.

[7] R. J. DiPerna, P. L. Lions, On the Cauchy problem for Boltzmann equations: Global existence and weak stability, Ann. of Math. 130 (1989), 321-366.

[8] R. Illner, T. Platkowski, Discrete velocity models of the Boltzmann equation: survey on the mathematical aspects of the theory, SIAM Rev. 30 (1988), 213-255.

[9] O. V. Ilyin, Symmetries, the current function, and exact solutions for Broadwell's twodimensional stationary kinetic model, Teoret. Mat. Fiz. 179 (2014), 350-359.

[10] A. N. Kolmogorov, Über Kompaktheit der Funktionenmengen bei der Konvergenz im Mittel, Nachr. Akad. Wiss. Göttingen Math.-Phys. KI. II 9 (1931), 60-63.

[11] M. Riesz, Sur les ensembles compacts de fonctions sommables, Acta Sci. Math. (Szeged) 6 (1933), 136-142. 